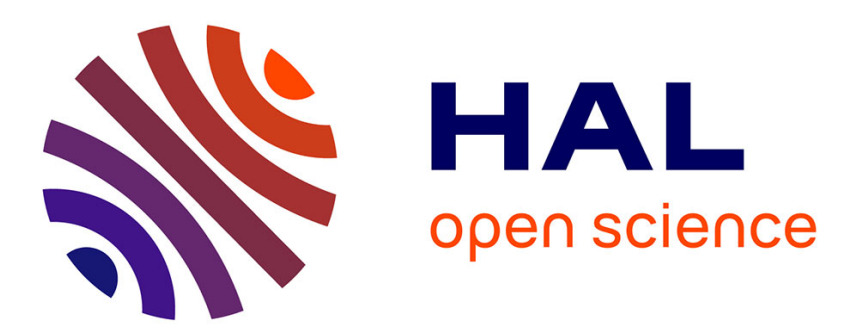

\title{
Mécanismes d'usure des outils coupants en usinage à sec de l'alliage de titane aéronautique Ti-6Al-4V
}

Mohammed Nouari, Madalina Calamaz, Franck Girot

\section{To cite this version:}

Mohammed Nouari, Madalina Calamaz, Franck Girot. Mécanismes d'usure des outils coupants en usinage à sec de l'alliage de titane aéronautique Ti-6Al-4V. Comptes Rendus Mécanique, 2008, 336 (10), pp.772-781. 10.1016/j.crme.2008.07.007 . hal-01018077

\section{HAL Id: hal-01018077 https://hal.science/hal-01018077}

Submitted on 3 Jul 2014

HAL is a multi-disciplinary open access archive for the deposit and dissemination of scientific research documents, whether they are published or not. The documents may come from teaching and research institutions in France or abroad, or from public or private research centers.
L'archive ouverte pluridisciplinaire HAL, est destinée au dépôt et à la diffusion de documents scientifiques de niveau recherche, publiés ou non, émanant des établissements d'enseignement et de recherche français ou étrangers, des laboratoires publics ou privés. 


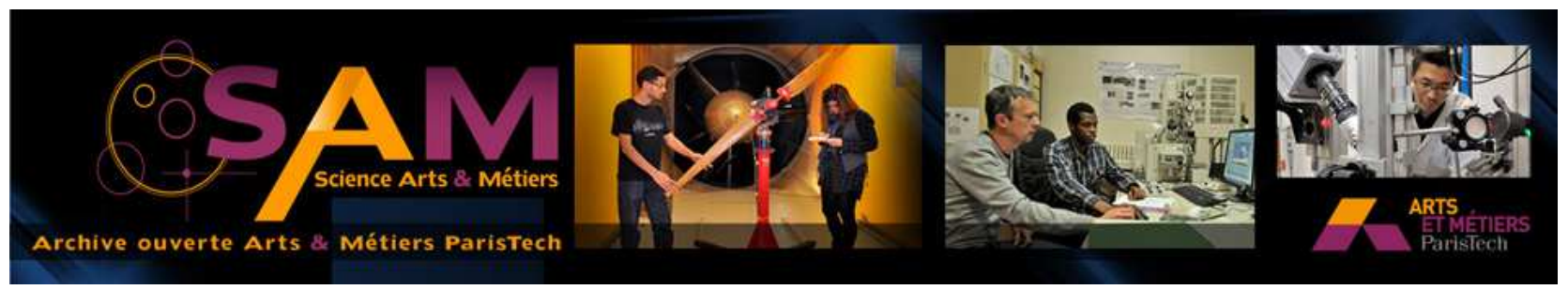

\section{Science Arts \& Métiers (SAM)}

is an open access repository that collects the work of Arts et Métiers ParisTech researchers and makes it freely available over the web where possible.

This is an author-deposited version published in: http://sam.ensam.eu

Handle ID: .http://hdl.handle.net/10985/8321

\section{To cite this version :}

Mohammed NOUARI, Madalina CALAMAZ, Franck GIROT - Mécanismes d'usure des outils coupants en usinage à sec de l'alliage de titane aéronautique Ti-6Al-4V - Comptes Rendus Mécanique - Vol. 336, nº10, p.772-781 - 2008 


\title{
Mécanismes d'usure des outils coupants en usinage à sec de l'alliage de titane aéronautique Ti-6Al-4V
}

\author{
Mohammed Nouari ${ }^{\mathrm{a}, *}$, Madalina Calamaz ${ }^{\mathrm{b}}$, Franck Girot ${ }^{\mathrm{b}}$ \\ a Laboratoire d'énergétique et de mécanique théorique et appliquée, LEMTA CNRS-UMR 7563, École nationale supérieure des mines de Nancy \\ (ENSMN), GIP-InSIC, 27, rue d'Hellieule, 88100 Saint-Dié-des-Vosges, France \\ ${ }^{\mathrm{b}}$ Laboratoire matériaux endommagement fiabilité ingénierie des procédés (LAMEFIP) ENSAM CER Bordeaux, esplanade des arts et métiers, \\ 33405 Talence cedex, France
}

Présenté par André Zaoui

\begin{abstract}
Résumé
Dans cette étude, l'alliage Ti-6Al-4V a été usiné à sec en configuration de c oupe orthogonale. Trois g éométries d'outils $\left(0^{\circ}, 15^{\circ}\right.$ et $\left.30^{\circ}\right)$, trois vitesses de coupe $(15,30$ et $60 \mathrm{~m} / \mathrm{min})$, et trois avances $(0,1,0,2$ et $0,3 \mathrm{~mm})$ ont été sélectionnées pour étudier les modes de dégradation des outils coupants. Les résultats montrent que l'effondrement d'arête est le principal mode d'usure des outils ayant un angle de coupe important $\left(30^{\circ}\right)$. L'analyse des paramètres tribologiques (frottement, température, pression de contact) a permis de mettre en évidence l'effet des paramètres d'usinage et de la géométrie d'outil sur les modes de dégradation pendant l'usinage. Pour citer cet article: M. Nouari et al., C. R. Mecanique 336 (2008).
\end{abstract}

\section{Abstract}

Wear mechanisms of cutting tools used in the dry machining of the aeronautic titanium alloy, Ti-6Al-4V. In the present study, the titanium alloy ' $\mathrm{Ti}-6 \mathrm{Al}-4 \mathrm{~V}$ ' is orthogonally machined without cutting fluid. During experiments, the cemented carbide tools were rigidly mounted on the tool holder with rake angles of $0^{\circ}, 15$ and $30^{\circ}$. Three cutting speeds of 15,30 and $60 \mathrm{~m} / \mathrm{min}$ and three feeds of $0.1,0.2$, and $0.3 \mathrm{~mm}$ were selected. The experimental results show that the tool catastrophic failure occurs just after the first pass of cutting for all cutting parameters when using the tool with a rake angle of $30^{\circ}$. The analysis of the tribological parameters (friction, temperature, contact pressure) shows the effect of the machining parameters and the tool geometry on the damage modes during dry machining. To cite this article: M. Nouari et al., C. R. Mecanique 336 (2008).

Mots-clés : Endommagement ; Usinage à sec du Ti-6Al-4V; Analyse par éléments finis ; Paramètres tribologiques ; Modes d'endommagement

Keywords: Damage; Dry machining, Ti-6Al-4V; Finite Element Analysis; Tribological parameters; Degradation modes

\footnotetext{
* Auteur correspondant.

Adresse e-mail : mohammed.nouari@ insic.fr (M. Nouari).
} 


\section{Introduction}

L'utilisation des alliages de titane est très répandue dans l'industrie aéronautique pour des applications exigeant une excellente résistance mécanique à haute température. Toutefois, ces alliages sont aussi connus pour être des matériaux difficiles à usiner. Les principaux problèmes rencontrés lors de l'usinage à sec des alliages de titane sont les faibles taux d'enlèvement de matière et les durées de vie d'outils très limitées. La faible conductivité thermique (de l'ordre de $15 \mathrm{~W} / \mathrm{m} \mathrm{K}$ ) et l'affinité chimique élevée pour beaucoup de matériaux causent souvent la formation d'une couche d'adhésion sur la face de l'outil provoquant ainsi une usure prématurée de celui-ci. Aussi, le faible module élastique du titane (50\% plus faible que celui de l'acier) est la cause principale des vibrations engendrées lors de l'usinage. En général, l'usinage des alliages de titane se fait souvent avec l'utilisation de la lubrification. Dans cette étude la possibilité de réaliser un usinage à sec est étudiée pour palier aux problèmes écologiques, sanitaires et économiques dans le milieu industriel. Dans un premier temps, l'étude propose d'analyser l'évolution des efforts de coupe lors de la coupe sans lubrification du Ti-6Al-4V. Les modes de dégradation des plaquettes sont ensuite identifiés à l'aide de la microscopie électronique à balayage $(\mathrm{MEB})$ et au profilomètre à lumière blanche. La simulation numérique de l'évolution des paramètres tribologiques (pression et température à l'interface) a permis de caractériser la nature du contact outil-copeau et d'expliquer le mode de dégradation mis en jeu.

\section{Mise en place des essais d'usinage à sec}

Les essais ont été réalisés à l'aide d'une raboteuse possédant une course maximale de $2 \mathrm{~m}$ et permettant de faire varier les vitesses de coupe et de retour de 6 à $60 \mathrm{~m} / \mathrm{min}$, (voir Fig. 1). La surface de la pièce usinée a été préparée en réalisant des rainures de largeur $4 \mathrm{~mm}$ (voir Fig. 2). Le matériau constituant la pièce est l'alliage de titane Ti-6Al-4V dont la composition chimique, les propriétés mécaniques et thermiques sont illustrées par les Tableaux 1 et 2. Lors d'une opération d'usinage, les outils de coupe sont soumis à des sollicitations mécaniques et thermiques extrêmes au voisinage de l'arête de coupe. Ceci est principalement dû à la faible usinabilité des alliages de titane, [1]. Les matériaux d'outils employés doivent donc posséder une dureté à chaud très élevée afin de résister aux hautes températures générées pendant la coupe. La majeure partie des matériaux d'outils perdent leurs duretés à haute température, ceci conduit à la rupture des liaisons entre les particules de carbure constituant l'outil et par conséquent à la dégradation et à l'usure de celui-ci.

Les outils de coupe considérés dans cette étude sont en carbure de tungstène non revêtu de nuance H13A (WC-6\% Co, type K20). L'angle de dépouille a été fixé à $12^{\circ}$ dans tous les cas d'essais. Trois corps d'outils ont été conçus spécialement pour obtenir des angles de coupe de $0^{\circ}, 15^{\circ}$ et $30^{\circ}$. Les angles positifs assurent une coupe stable et une action efficace de l'arête de coupe de l'outil. Donyo [2] a confirmé que les angles positifs dans le cas des outils carbures facilitent l'écoulement du copeau grâce notamment à la formation des couches d'adhésion constituées de dépôt de particules de titane pur. Les angles de coupe nulles ou négatives ne facilitent pas la formation de ces dépôts

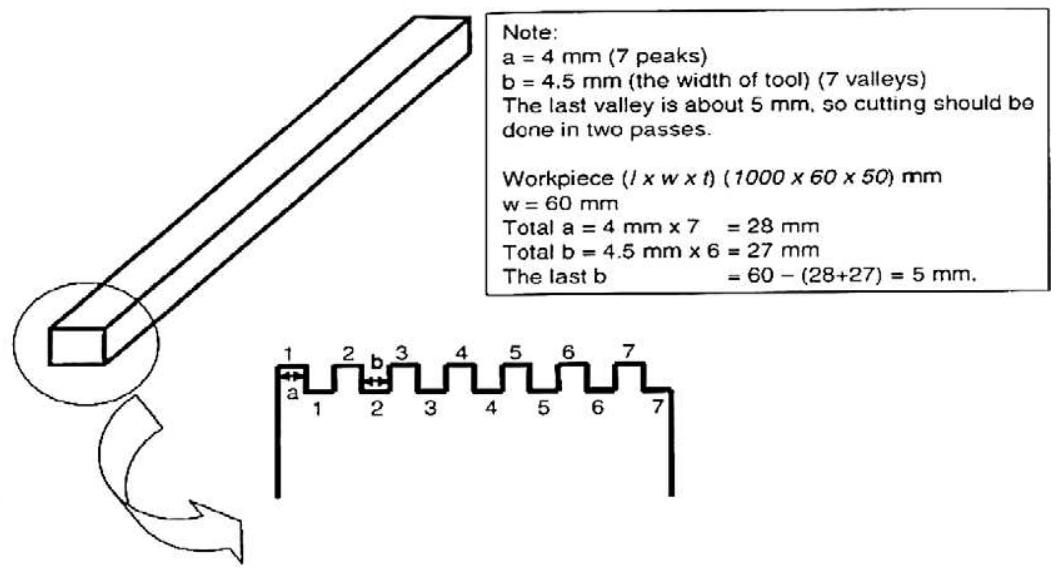

Fig. 1. Géométrie de la pièce usinée ; $a=4 \mathrm{~mm}$ et $b=4.5 \mathrm{~mm}$. 


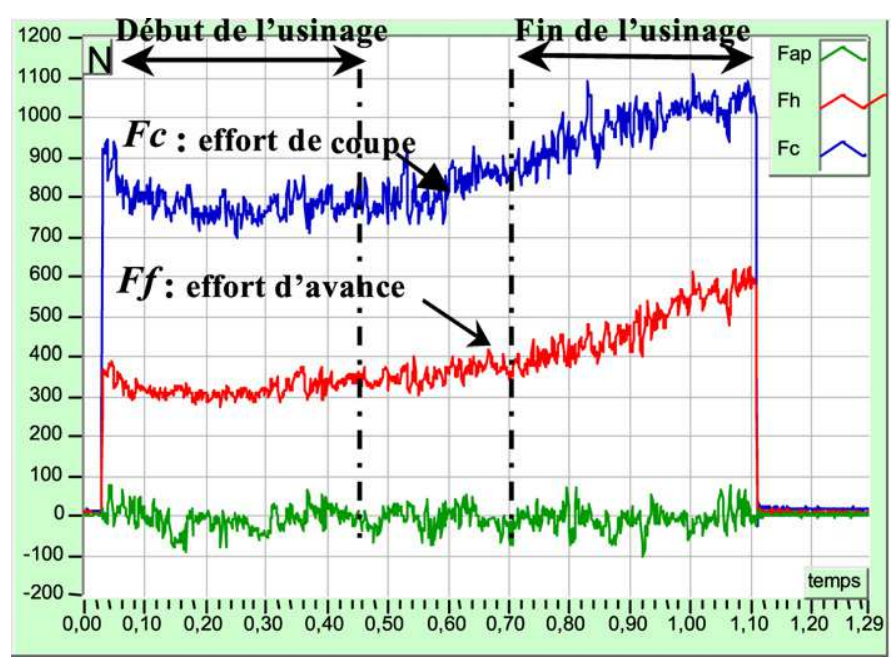

(a)

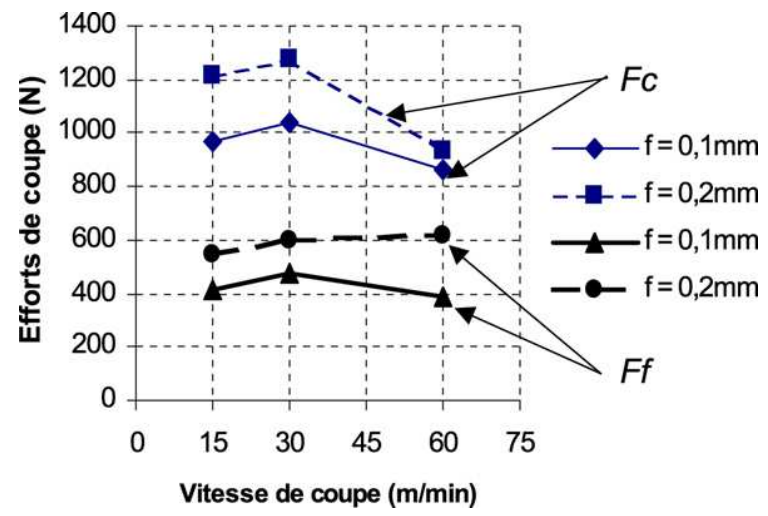

(b)

Fig. 2. Evolution des efforts de coupe et d'avance. (a) En fonction du temps d'usinage pour les conditions : $V_{c}=60 \mathrm{~m} / \mathrm{min}, f=0,1 \mathrm{~mm} ; \alpha=0^{\circ}$. (b) En fonction de la vitesse de coupe $V_{c}$ et de l'avance $f$.

Tableau 1

Propriétés mécaniques et thermiques de l'alliage Ti-6Al-4V

\begin{tabular}{lllllllll}
\hline $\begin{array}{l}\text { Résistance } \\
\text { à la traction } \\
(\mathrm{MPa})\end{array}$ & $\begin{array}{l}\text { Limite } \\
\text { élastique } \\
(\mathrm{MPa})\end{array}$ & $\begin{array}{l}\text { Elongation } \\
(\%)\end{array}$ & $\begin{array}{l}\text { Réduction } \\
\text { d'aire }(\%)\end{array}$ & $\begin{array}{l}\text { Module } \\
\text { élastique } \\
(\mathrm{GPa})\end{array}$ & $\begin{array}{l}\text { Dureté } \\
(\mathrm{HV})\end{array}$ & $\begin{array}{l}\text { Densité } \\
\left(\mathrm{g} / \mathrm{cm}^{3}\right)\end{array}$ & $\begin{array}{l}\text { Chaleur spécifique } \\
20-100^{\circ} \mathrm{C} \\
(\mathrm{J} / \mathrm{kg} \mathrm{K})\end{array}$ & $\begin{array}{l}\text { Conductivité } \\
\text { thermique à 20 }\end{array}$ \\
\hline 931 & 862 & 10 & 25 & 110 & 340 & 4,43 & 580 & 7,3 \\
\hline
\end{tabular}

Tableau 2

Composition chimique de l'alliage Ti-6Al-4V (wt. \%)

\begin{tabular}{lllllll}
\hline $\mathrm{Fe}$ & $\mathrm{V}$ & $\mathrm{Al}$ & $\mathrm{C}$ & $\mathrm{O}$ & $\mathrm{N}$ & $\mathrm{O}+2 \mathrm{~N}$ \\
\hline Max 0,25 & $3,5-4,5$ & $5,5-6,75$ & Max 0,8 & Max 0,2 & Max 0,3 & Max 0,25 \\
\hline
\end{tabular}

qui protègent en général l'arête de coupe. En revanche, une géométrie avec des angles de coupe négatifs présente l'avantage de donner une meilleure robustesse à l'arête de coupe.

Les vitesses de coupe et les avances choisies dans cette étude sont respectivement : 15, 30 et $60 \mathrm{~m} / \mathrm{min}, 0,1,0,2$ et $0,3 \mathrm{~mm}$. Tous les essais ont été réalisés sans lubrification (usinage à sec). Plusieurs raisons sont à la base du choix du matériau d'outil. Parmi les 7 matériaux couramment utilisés : fonte, acier rapide, carbure, céramique, CBN (Nitrure 


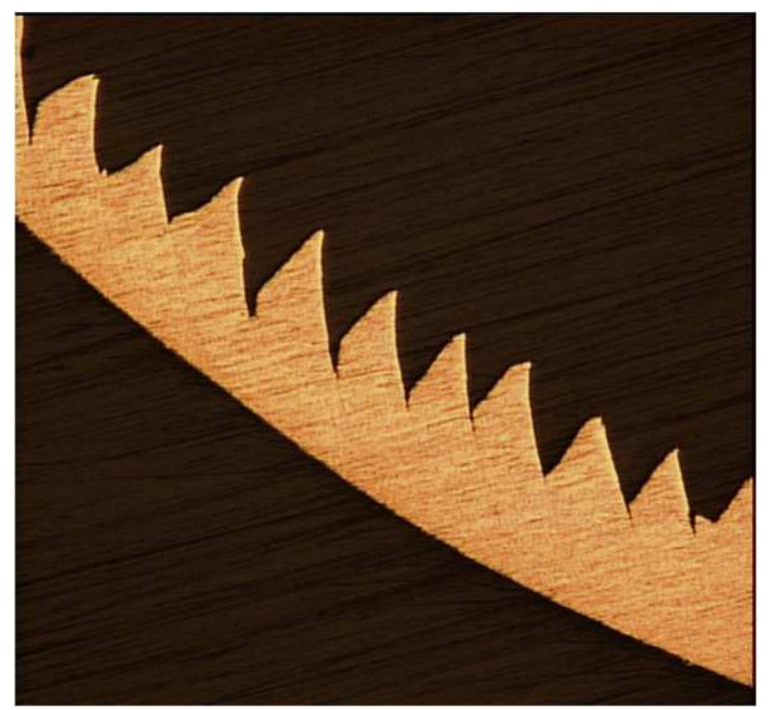

(a)

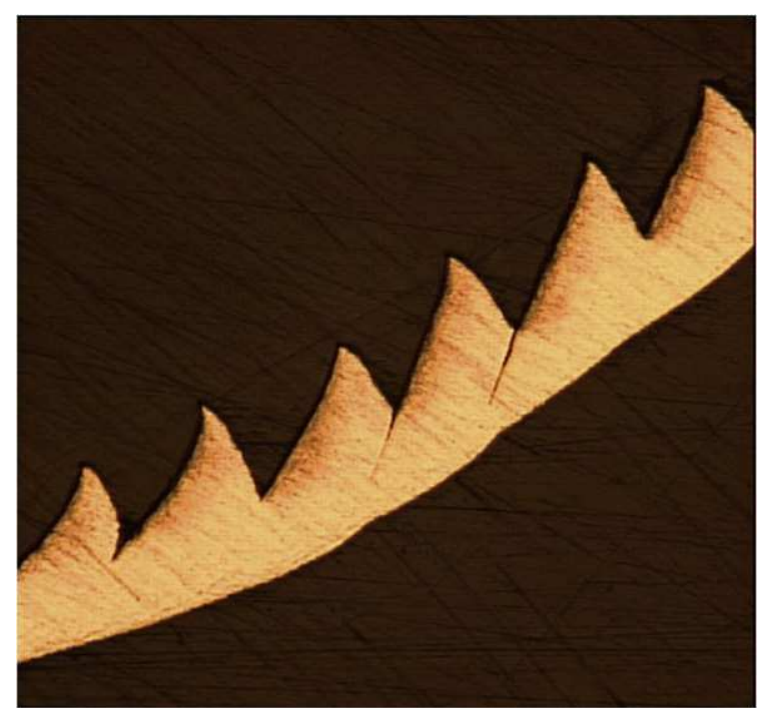

(b)

Fig. 3. Morphologie des copeaux obtenus en coupe orthogonale à sec de l'alliage Ti-6Al-4V. (a) Copeau obtenu avec $V_{c}=30 \mathrm{~m} / \mathrm{min},(\mathrm{b})$ avec $V_{c}=60 \mathrm{~m} / \mathrm{min}$. Les autres conditions de coupe sont analogues dans les deux cas : angle de coupe $\alpha=0^{\circ}$, avance $f=0,3 \mathrm{~mm}$.

de Bore Cubique), diamant, alliages de cobalt, les carbures sont les plus recommandés pour l'usinage des alliages de titane [3-5]. La fonte, l'acier rapide et l'alliage de cobalt sont déconseillés à cause de leur faible résistance aux chocs à des températures élevées et les déformations plastiques sévères qu'ils engendrent. Les outils céramiques ne sont pas recommandés pour la coupe des alliages de titane à cause de leur faible performance due à la réactivité chimique avec le titane (usure prématurée). De plus, ils sont sensibles aux chocs mécaniques et thermiques à cause de leur ténacité réduite [6]. Les matériaux super durs (CBN) et le diamant polycristallin (PCD)) ont montré une bonne performance en termes de taux d'usure dans l'usinage du titane. Ces types d'outils permettent des vitesses de coupe de l'ordre de $150 \mathrm{~m} / \mathrm{min}$, mais leur utilisation engendre des coûts trop élevés pour les applications industrielles [7]. Les alliages de titane sont aussi usinés avec des outils CBN dans des conditions de finition à des vitesses maximales de $350 \mathrm{~m} / \mathrm{min}$, des avances de l'ordre de $0.05 \mathrm{~mm} / \mathrm{min}$ et profondeurs de coupe de $0,5 \mathrm{~mm}$ [8].

Dearnley et al. $[9,10]$ ont montré que les carbures non-revêtus de grade $\mathrm{K}$ présentent le meilleur choix pour la coupe des alliages de titane. Ils suggèrent l'utilisation des carbures à $6 \%$ de Cobalt avec une taille des grains de WC de 0,8 à $1,4 \mu \mathrm{m}$ qui donne une meilleure performance.

Par ailleurs, les revêtements tels que : TiC, TiCN, TiN-TiC, $\mathrm{Al}_{2} \mathrm{O}_{3}-\mathrm{TiC}$, TiN-Ti(C,N)-TiC, $\mathrm{Al}_{2} \mathrm{O}_{3}, \mathrm{TiB}_{2}$ ont montrés des taux d'usure beaucoup plus importants que les carbures sans revêtement [1].

\section{Evolution des paramètres de coupe en usinage à sec des alliages Ti-6Al-4V}

\subsection{Efforts de coupe et nature du contact outil-copeau}

L'évolution des efforts de coupe $\left(F_{c}\right)$ et d'avance $\left(F_{f}\right)$ pendant la coupe orthogonale est mise en évidence par la Fig. 3 pour la condition de coupe $\left(V_{c}=60 \mathrm{~m} / \mathrm{min}, f=0,1 \mathrm{~mm}\right)$ et une géométrie d'outil à $0^{\circ}$. Une augmentation brutale des efforts est particulièrement notée au-delà de $60 \mathrm{~ms}$ d'usinage, montrant ainsi l'évolution de l'usure de la plaquette entre le début et la fin de l'opération d'usinage. Cette usure est présente à la fois au niveau de la face de coupe et de dépouille de l'outil. La Fig. 3(b) illustre l'évolution des efforts en fonction de la vitesse de coupe et de l'avance. Une légère augmentation est observée entre 15 et $30 \mathrm{~m} / \mathrm{min}$ et puis une chute au delà $(16 \%$ pour $f=0,1 \mathrm{~mm}$ et $27 \%$ pour $f=0,2 \mathrm{~mm}$ ). Cette décroissance est due à l'adoucissement du matériau provoqué par l'augmentation importante de la température pendant l'usinage à sec. L'adoucissement de l'alliage de titane conduit principalement à la segmentation des copeaux et à des longueurs de contact entre l'outil et le copeau plus faibles comme le montre la Fig. 4. 


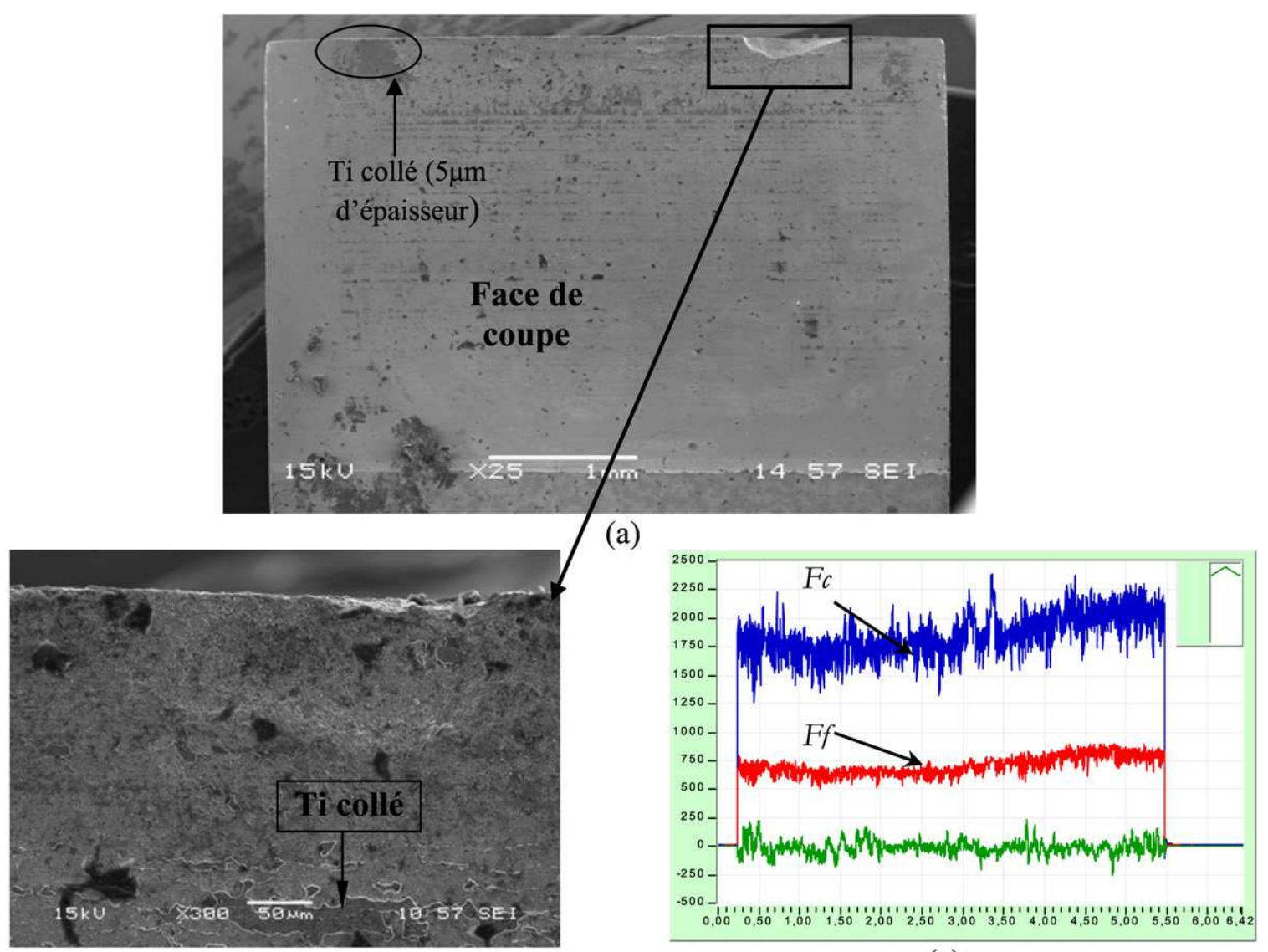

(b)

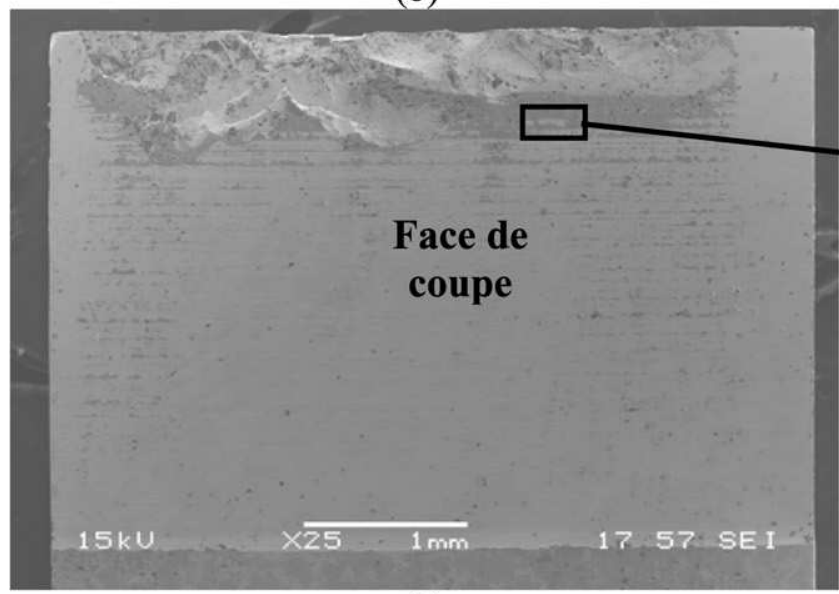

(c)

(d)

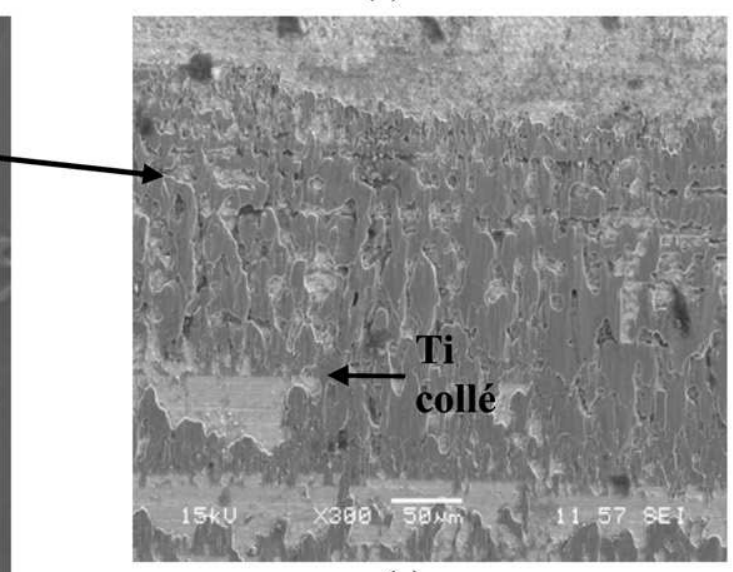

(e)

Fig. 4. Micrographies des surfaces d'outils usées : (a), (b) et (c) $V_{c}=15 \mathrm{~m} / \mathrm{min}$, (d) et (e) $V_{c}=60 \mathrm{~m} / \mathrm{min} ; f=0.3 \mathrm{~mm}$, $1^{\prime}$ angle de coupe $\alpha=0^{\circ}$.

Les coefficients de frottement apparents ont été calculés à partir des efforts de coupe enregistrés lors des essais d'usinage, [11,12],

$$
\mu_{0}=\frac{F_{t}}{F_{n}}=\frac{F_{f}+F_{c} \tan \alpha}{F_{c}-F_{f} \tan \alpha}
$$


Tableau 3

Evolution du coefficient de frottement à sec à l'interface outil-copeau pour différentes conditions de coupe

\begin{tabular}{|c|c|c|c|c|c|c|c|c|c|c|}
\hline $\begin{array}{l}\text { Essai } \\
\mathrm{n}^{\circ}\end{array}$ & $\begin{array}{l}V_{c} \\
(\mathrm{~m} / \mathrm{min})\end{array}$ & $\begin{array}{l}\alpha \\
\left({ }^{\circ}\right)\end{array}$ & $\begin{array}{l}f \\
(\mathrm{~mm})\end{array}$ & $\begin{array}{l}F_{c} \\
\text { (début) }\end{array}$ & $\begin{array}{l}F_{c} \\
\text { (end) }\end{array}$ & $\begin{array}{l}F_{f} \\
\text { (début) }\end{array}$ & $\begin{array}{l}F_{f} \\
\text { (fin) }\end{array}$ & $\begin{array}{l}\mu \\
\text { (début) }\end{array}$ & $\begin{array}{l}\mu \\
\text { (fin) }\end{array}$ & Modes d'endommagement \\
\hline 1 & 15 & 0 & 0,1 & 900 & 1100 & 400 & 480 & 0,44 & 0,43 & \\
\hline 2 & 15 & 0 & 0,2 & 1200 & 1350 & 520 & 600 & 0,43 & 0,44 & Usure normale \\
\hline 3 & 15 & 0 & 0,3 & 1750 & 2100 & 700 & 850 & 0,4 & 0,4 & \\
\hline 4 & 30 & 0 & 0,1 & 970 & 1200 & 450 & 550 & 0,46 & 0,46 & - \\
\hline 5 & 30 & 0 & 0,2 & 1250 & 1420 & 580 & 1200 & 0,46 & 0,85 & fissuration + adhésion + écaillage \\
\hline 6 & 30 & 0 & 0,3 & 1300 & 1790 & 420 & 700 & 0,32 & 0,39 & - \\
\hline 7 & 60 & 0 & 0,1 & 800 & 1000 & 300 & 600 & 0,37 & 0,6 & - \\
\hline 8 & 60 & 0 & 0,2 & 900 & 1100 & 580 & 710 & 0,64 & 0,65 & - \\
\hline 9 & 60 & 0 & 0,3 & 1600 & 1950 & 800 & 1170 & 0,5 & 0,6 & - \\
\hline 10 & 15 & 30 & 0,1 & 450 & 600 & 60 & 50 & 0,77 & 0,7 & Usure catastrophique \\
\hline
\end{tabular}

Avec un angle de coupe nul, la relation (1) se réduit à :

$$
\mu_{0}=\frac{F_{f}}{F_{c}}
$$

La décroissance du frottement à l'interface outil-copeau au début d'usinage quand la vitesse de coupe $V_{c}$ augmente, peut aussi faire diminuer les efforts quand l'usure n'est pas trop prononcée. En fait, Moufki et al. [13] ont montré que le frottement obéit à une loi souvent dépendante de la température de coupe :

$$
\mu_{0}=\mu_{0}\left(T_{c}\right)
$$

L'augmentation de la température provoquée par l'augmentation de la vitesse de coupe engendre une diminution du coefficient de frottement. Ceci se traduit par un changement de la nature du contact (passage d'un contact collant à un contact glissant) et par conséquent la diminution des efforts de coupe.

A la fin de l'opération de coupe et comme il a été remarqué lors des essais d'usinage, le frottement augmente et les conditions de contact changent. Ainsi les efforts augmentent de façon importante. Le frottement moyen à l'interface est donc un moyen d'évaluation efficace de la dégradation de la surface de l'outil. Le Tableau 3 montre le changement du frottement en fonction des conditions de coupe considérées dans l'étude.

\subsection{Modes et mécanismes d'endommagement des outils de coupe}

Les analyses au profilomètre optique et au MEB relatives aux essais 1, 2 et 3 du le Tableau 3 montrent une usure normale des plaquettes (voir Figs. 5(a) et 5(b)). En fait, des constituants de l'alliage de titane (Ti, $\mathrm{Al}, \ldots$ ) ont été détectés sur la face de coupe ainsi qu'une écaille de profondeur égale à $17 \mu \mathrm{m}$. La plaquette n'a pas subi une usure importante et l'évolution des efforts de coupe lors de l'essai est restée normale (voir Fig. 5(c)). Les Figs. 5(d) et 5(e) montrent des micrographies de la plaquette correspondant à l'essai $n^{\circ} 9$. Une augmentation de la quantité de titane collé est notée en comparaison avec la plaquette de l'essai $\mathrm{n}^{\circ} 3$; ceci est la conséquence directe de l'élévation de la température à l'interface outil-copeau quand la vitesse de coupe augmente.

Les résultats de la simulation numérique de la Fig. 6(a) montrent que la température à l'interface a presque doublé pour une augmentation de la vitesse de coupe de 15 à $60 \mathrm{~m} / \mathrm{min}$ (conditions 3 et 9 ). De plus, la pression au niveau de la pointe de l'outil croît également avec la vitesse $\left(P=1900 \mathrm{MPa}\right.$ pour $V_{c}=15 \mathrm{~m} / \mathrm{min}$ et $P=2500 \mathrm{MPa}$ pour $V_{c}=$ $60 \mathrm{~m} / \mathrm{min}$ (Fig. 6(b)). Les fortes pressions et les températures importantes à l'interface expliquent l'usure prononcée des outils de coupe dans le cas de la condition $\mathrm{n}^{\circ} 9$ (écaille de $115 \mu \mathrm{m}$ de profondeur).

Parmi les essais 4, 5 et 6 , seul l'outil correspondant à la condition 5 présente une usure importante. Lors de cet essai, en plus du collage de l'alliage de titane sur la plaquette et de l'écaillage de la face de coupe de l'outil, une fissuration de l'arête a également été observée sur la micrographie de la Fig. 7(a). De plus, une augmentation brutale de l'effort d'avance $\left(F_{f}\right)$ à la fin de l'essai est remarquée sur la Fig. 7(c). Ceci est dû à l'arrachement du matériau de la plaquette sur la face de dépouille (voir Fig. 7(b)). Cet écaillage important de la face de coupe et de dépouille explique l'augmentation brutale du coefficient de frottement entre le début $(\mu=0.46)$ et la fin $(\mu=0.85)$ de l'usinage sous cette même condition de coupe (voir Tableau 3). 


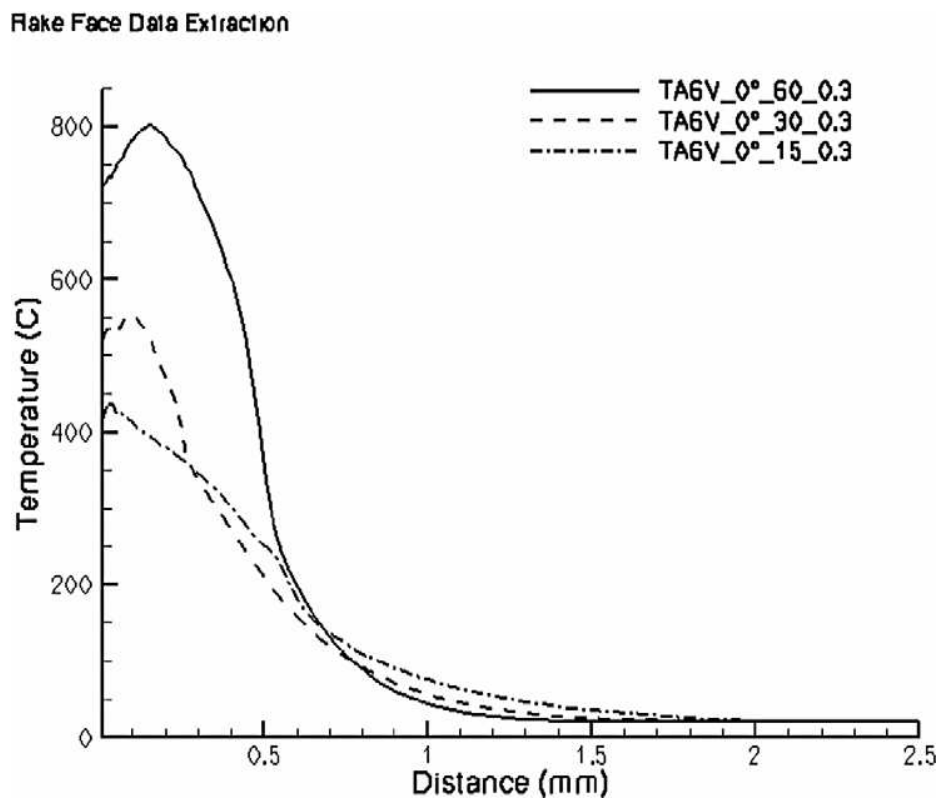

(a)

Fake Face Dala Exiracion

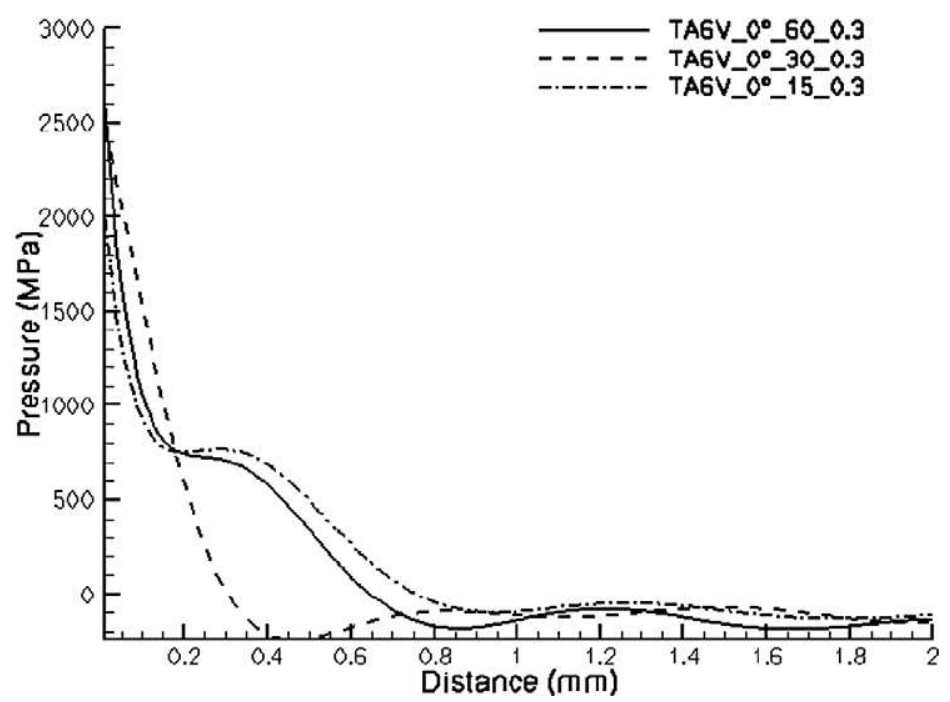

(b)

Fig. 5. (a) Evolution de la température à l'interface. (b) Evolution de la pression de contact pour trois vitesses de coupe $V_{c}=15,30$ et $60 \mathrm{~m} / \mathrm{min}$, l'avance est fixée à $0,3 \mathrm{~mm}$.

Comme le montre les Figs. 7(d) et 7(e) la condition de coupe $\mathrm{n}^{\circ} 10$ a donné lieu à une usure catastrophique de la plaquette caractérisée par un recul important de l'arête (de 0,5 mm) et une écaille de $390 \mu \mathrm{m}$ de profondeur. En effet, un effondrement d'arête a été systématiquement remarqué pour tous les essais réalisés avec des outils ayant un angle de coupe important $\left(\alpha=30^{\circ}\right)$. Les faibles valeurs des efforts de coupe enregistrés $\left(F_{c}=450 \mathrm{~N}\right.$ et $\left.F_{f}=60 \mathrm{~N}\right)$ n'expliquent pas cette usure catastrophique. Afin d'expliquer cet effondrement d'arête, une analyse par élément finis a été réalisée pour calculer les différentes paramètres tribologiques (pression, température de coupe, etc.).

En coupe des métaux, les résultats montrent souvent que plus l'angle de coupe est grand, plus l'angle de cisaillement est important; ainsi l'écoulement du copeau est facilité et les efforts de coupe sont généralement réduits. Cependant, ces tendances ne sont pas reproduites dans le cas de l'usinage à sec de l'alliage aéronautique Ti-6Al-4V. 


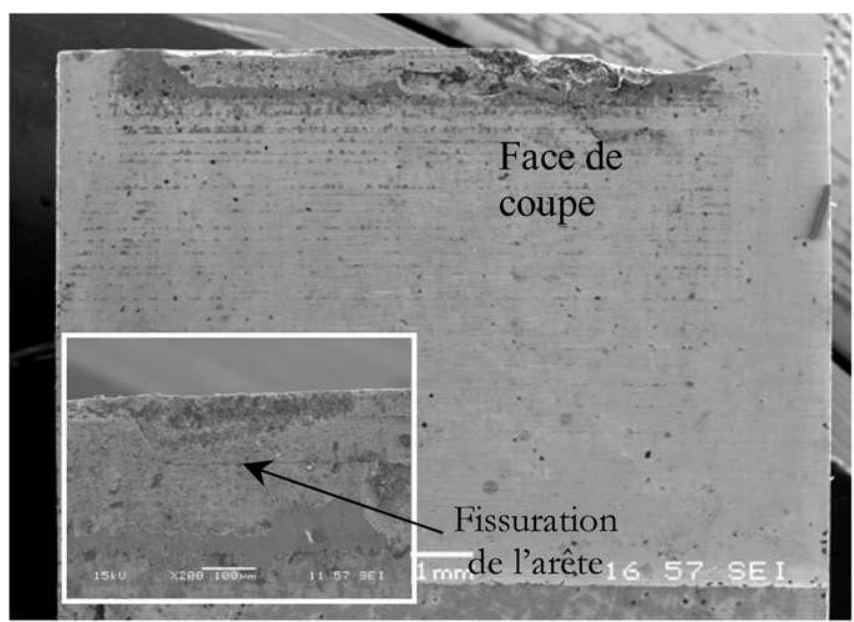

(a)
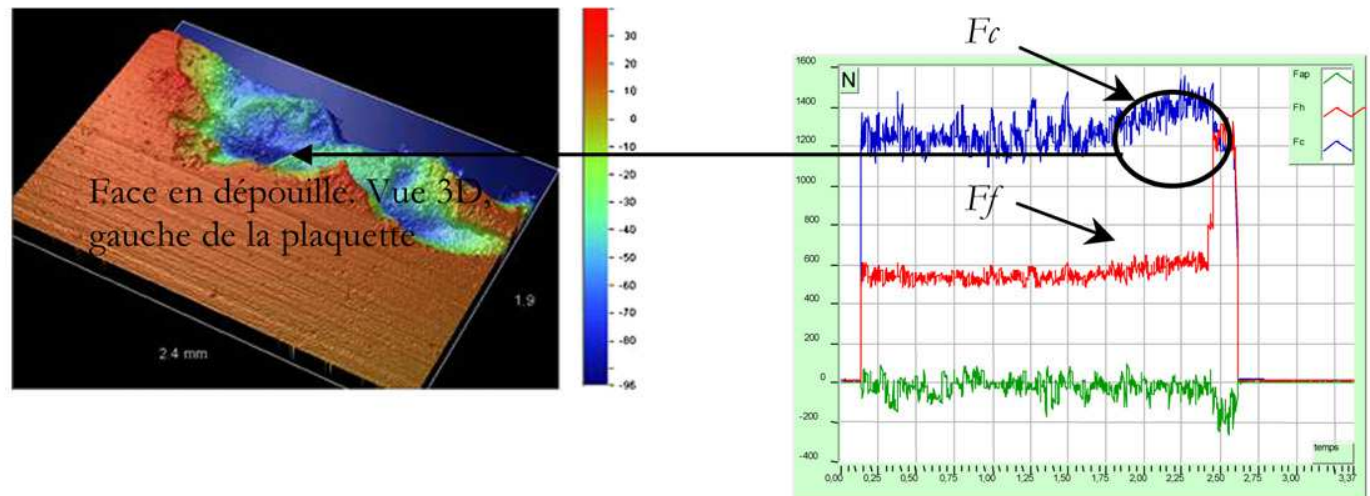

(b)

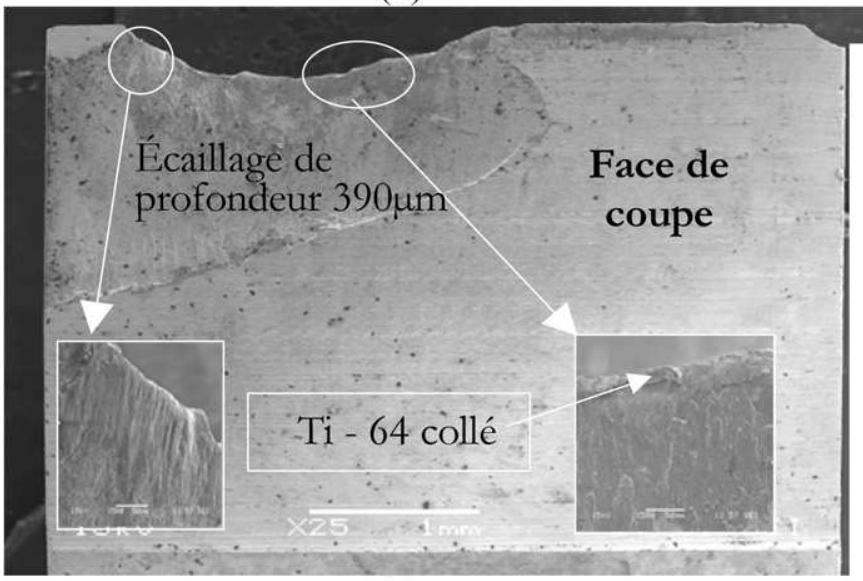

(d) (c)

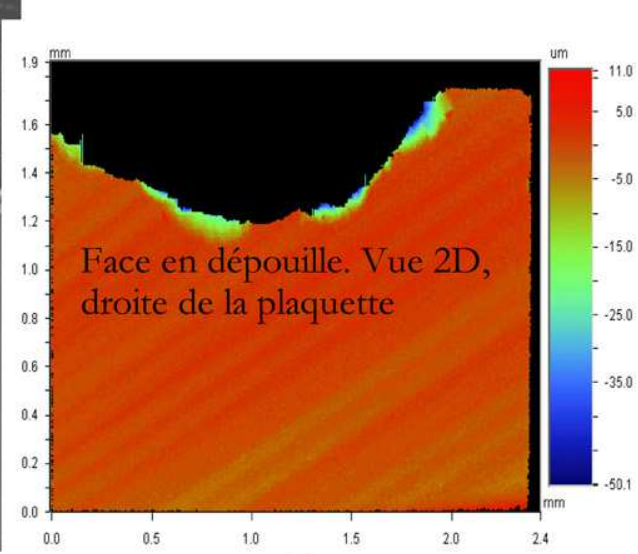

(e)

Fig. 6. Images MEB et profilomètre des plaquettes : (a), (b) et (c) $V_{c}=30 \mathrm{~m} / \mathrm{min}$, avance $=0.2 \mathrm{~mm}$, angle de coupe $=0^{\circ} ;(\mathrm{d})$ et (e) $V_{c}=15 \mathrm{~m} / \mathrm{min}$, avance $=0.1 \mathrm{~mm}$, angle de coupe $=30^{\circ}$.

Les résultats de la simulation numérique, illustrés dans la Fig. 7 montre que la température à l'interface outil-copeau est du même ordre pour les plaquettes à $0^{\circ}$ et $30^{\circ}$. Celle-ci est principalement distribuée au voisinage de l'arête de coupe. De plus, sous l'effet des hautes pressions (de l'ordre de 2,5 GPa) et sous l'effet du frottement dynamique 


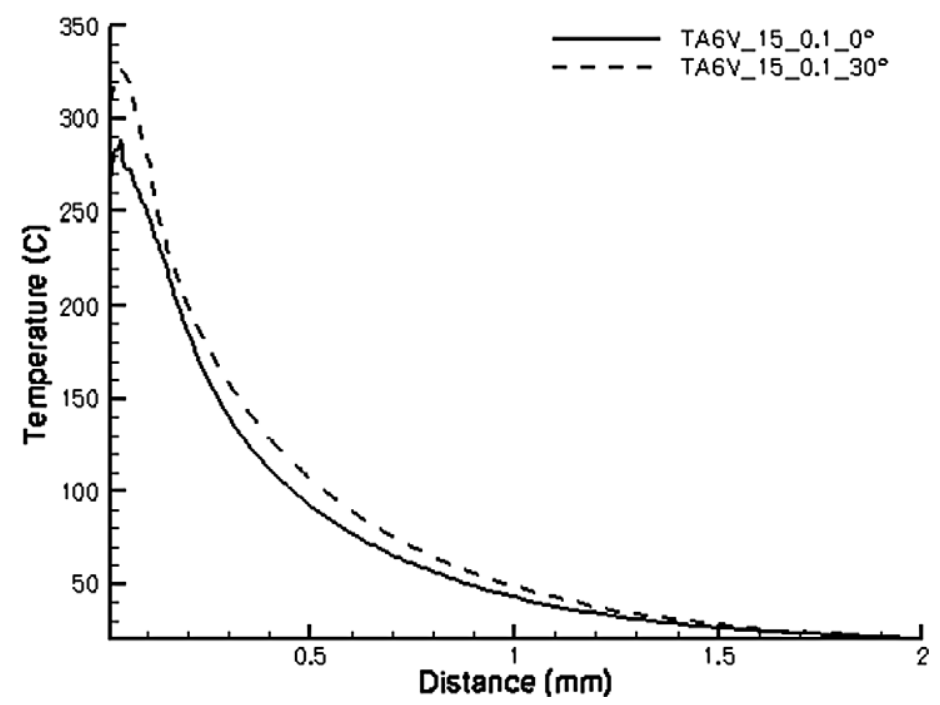

(a)

Rake Face Dala Exiraction

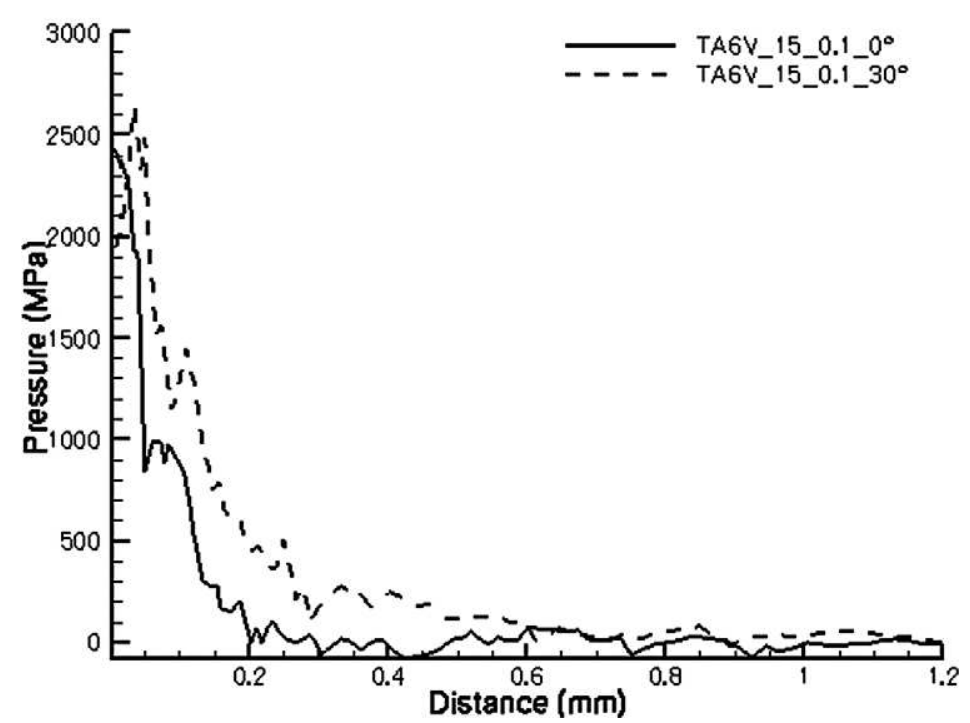

(b)

Fig. 7. Evolution des paramètres tribologiques pour deux géométries différentes $0^{\circ}$ et $30^{\circ}$. La vitesse de coupe $V_{c}=15 \mathrm{~m} / \mathrm{min}$ et $1^{\prime}$ avance $f=0.1 \mathrm{~mm}$. (a) Température de coupe. (b) Pression de contact.

intense à l'interface, l'arête s'effondre (conditions de chargement extrêmes). Ceci explique la principale cause de la rupture catastrophique et de l'effondrement d'arête des outils ayant un angle de coupe assez grand.

\section{Conclusions}

Les résultats présentés dans cette étude montrent que les efforts de coupe ne permettent pas de décrire en temps réel l'évolution de l'usure des outils en fonction des paramètres d'usinage (géométrie des outils, conditions de coupe, etc.). Afin d'expliquer les modes d'endommagement des outils pendant l'usinage à sec du Ti-6Al-4V, il est indispensable de caractériser la nature du frottement ainsi que les différents paramètres contrôlant le contact outil-copeau (température, pression de contact, longueur de contact, etc.). La mesure de ces paramètres est souvent difficile à réa- 
liser expérimentalement, la simulation numérique semble être un bon moyen complémentaire pour relier l'usure aux différents paramètres tribologiques. Les résultats d'essais et de simulation numérique montrent que l'augmentation de la température et de la pression de contact en fonction de la vitesse de coupe expliquent la croissance rapide de la couche d'adhérence du titane ainsi que l'écaillage de la face de coupe de l'outil. La comparaison des résultats en termes de frottement, température et pression montre qu'une géométrie d'outil avec un angle de coupe trop grand est le principal responsable de l'usure catastrophique des outils.

\section{Références}

[1] E.O. Ezugwu, Z.M. Wang, Titanium alloys and their machinability - a review, J. Mater. Process. Technol. 68 (1997) $262-274$.

[2] Y.B. Donyo, Contribution à l'analyse des modes de formation du copeau en usinage grande vitesse du TA6V, Thèse de doctorat, École Centrale de Nantes et Université de Nantes, 2002.

[3] H.J. Siekmann, How to machine titanium, The Tool Engineer 34 (1955) 78-82.

[4] R. Komanduri, Some clarifications on the mechanics of chip formation when machining titanium alloys, Wear 76 (1982) 15-34.

[5] L.N. López de Lacalle, J. Perez-Bilbatua, J.A. Sanchez, J.I. Llorente, A. Gutierrez, J. Alboniga, Using high pressure coolant in the drilling and turning of low machinability alloys, Int. J. Adv. Manuf. Technol. 16 (2000) 85-91.

[6] E.O. Ezugwu, J. Bonney, Y. Yamane, An overview of the machinability of aeroengine alloys, J. Mater. Process. Technol. 134 (2003) $233-253$.

[7] E.O. Ezugwu, Key improvements in the machining of difficult-to-cut aerospace superalloys, Int. J. Machine Tools \& Manufacture 45 (2005) $1353-1367$.

[8] Z.A. Zoya, R. Krishnamurthy, The performance of CBN tools in the machining of titanium alloys, J. Mater. Process. Technol. 100 (2000) $80-86$.

[9] P.A. Dearnley, A.N. Grearson, Mater. Sci. Technol. 2 (1986) 47-58.

[10] P.A. Dearnley, A.N. Grearson, J. Aucote, High Tech. Ceram. 38 (1987) 2699-2712.

[11] E. Merchant, Mechanical of the metal cutting process I. Orthogonal cutting and a type 2 chip, J. Appl. Phys. 16 (1945) $267-275$.

[12] E. Merchant, Mechanical of the metal cutting process. Orthogonal cutting, J. Appl. Phys. 16 (1945) 318-324.

[13] A. Moufki, A. Molinari, D. Dudzinski, Modelling of orthogonal cutting with a temperature dependent friction law, J. Mech. Phys. Solids 46 (1998) 2103-2138. 